\title{
Comparison of heater architectures for thermal control of silicon photonic circuits
}

\author{
Adil Masood*, Marianna Pantouvaki ${ }^{\dagger}$, Guy Lepage ${ }^{\dagger}$, Peter Verheyen ${ }^{\dagger}$, Joris Van Campenhout ${ }^{\dagger}$, \\ Philippe Absil ${ }^{\dagger}$ and Dries Van Thourhout*, Wim Bogaerts*, \\ *Photonics Research Group, Ghent University - imec,Department of Information Technology \\ Center of Nano- and Biophotonics (NB-photonics), Ghent, Belgium \\ ${ }^{\dagger}$ IMEC vzw, Leuven, Belgium
}

\begin{abstract}
We present a comparison of integrated heaters for silicon photonics, based on doped silicon, silicide and tungsten metallization, with and without trenches and undercut for insulation. Results show similar thermo-optic efficiency, but with electrical resistivities spanning 2 orders of magnitude.
\end{abstract}

\section{INTRODUCTION}

One of the challenges for WDM with silicon photonics is thermal control. Silicon has a high thermo-optic coefficient, with wavelength shifts of $80 \mathrm{pm} / \mathrm{K}$. Resistive heaters for thermo-optic control of silicon waveguides have already been demonstrated in many variations [1], [2], [3]. We present oneto-one comparison between different resistive heaters within in the same silicon photonics technology platform: resistors in doped silicon, Ni-silicide, and Tungsten (W) wiring. These heaters have different line resistivities, making it possible to build heaters with very different dimensions that can still be directly driven from a low-voltage CMOS circuit. Heater efficiency is mainly determined by the phase that can be induced in the waveguide for a certain amount of dissipated electrical power. This is expressed in power (in $\mathrm{mW}$ ) needed for a $\pi$ phase shift. As a resistor converts all electrical power into heat, the efficiency of a thermo-optic tuner will be largely determined by the thermal environment: how much heat is transferred to the waveguide, and how much leaks to the surroundings. To minimize unwanted leakage of heat, we add thermal insulation trenches, including an undercut of the silicon substrate. We will discuss and compare the different types of heaters (silicon, silicide and metal) in thermo-optic and electrical performance and how the thermal insulation affects the heater performance in both static and dynamic way.

\section{DESIGN OF EXPERIMENT}

In a CMOS-like silicon photonics process different materials can be used for heaters. This is schematically depicted in Fig. 1 for imec's silicon photonics platform. A resistor can be made in in doped silicon, positioned next to the waveguide, or alternatively in a silicide line. Silicides are already used for contacting plasma-dispersion p-n modulators. For lower line resistivities, metals can be used. Because the heater lines should have a higher resistivity than the copper $(\mathrm{Cu})$ metallization, we use tungsten (W) [3] lines above the waveguide, which makes them suitable in situations where no space is available next to the waveguide. An alternative is using the waveguide itself as a resistor, by lightly doping it, but this will induce additional loss in the waveguide.

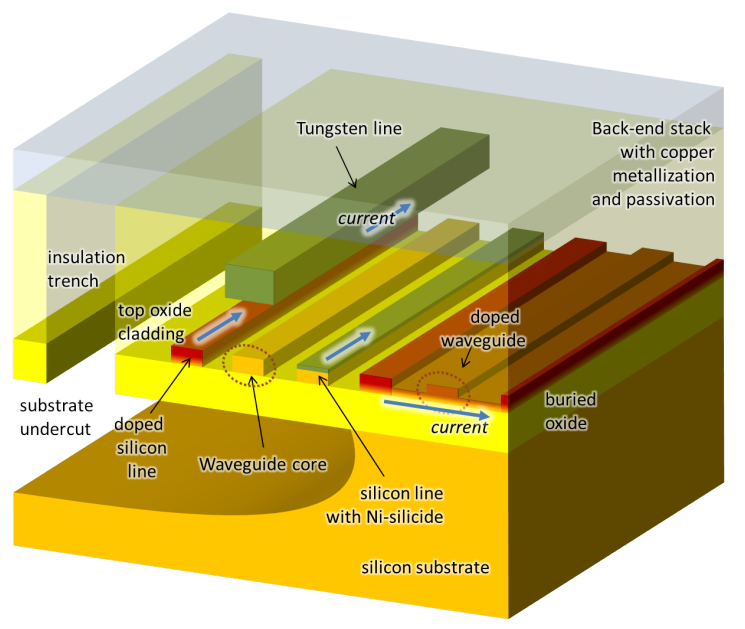

Fig. 1. Different heater architectures in imec's silicon photonics platform.

Our experiment is depicted in Fig. 2. We use an asymmetric Mach-Zehnder interferometer (MZI) with a free-spectral range (FSR) of approximately $13 \mathrm{~nm}$. One arm is flanked with 5 heaters with 3 different lengths $L$, and 3 different lateral offsets $s$. For each type of heater we implemented such an MZI. Each heater is equipped with 4-point probe pads, so the resistance can be accurately extracted. The different lengths allow us to separate the bulk effect from the edge effects.

\section{FABRICATION AND CHARACTERIZATION}

\section{A. Fabrication}

The devices were fabricated in imec's silicon photonics platform [4] on an SOI wafer with $220 \mathrm{~nm}$ silicon on a $2 \mu \mathrm{m}$ buried oxide. First, the passive waveguides are processed. Then dopants are implanted for modulators; the same implants are used for heaters. Using a patterned oxide mask, local silicidation is applied for contacting, as well as for heater lines. After oxide cladding deposition, contact holes are etched, filled with tungsten and planarized. Then, tungsten heaters are defined on top using a damascene process. Finally, we add a standard $\mathrm{Cu}$-damascene back-end and passivation. On some wafers, we processed thermal insulation trenches by deepetching down to the silicon substrate, after which an isotropic selective silicon etch was applied to the silicon substrate to undercut the waveguides. 


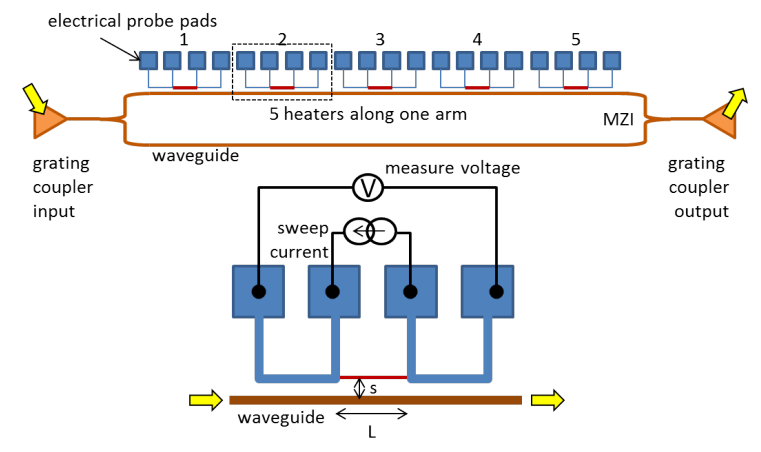

Fig. 2. Experiment for characterization of one type of heater. This design is replicated for the different heater types.
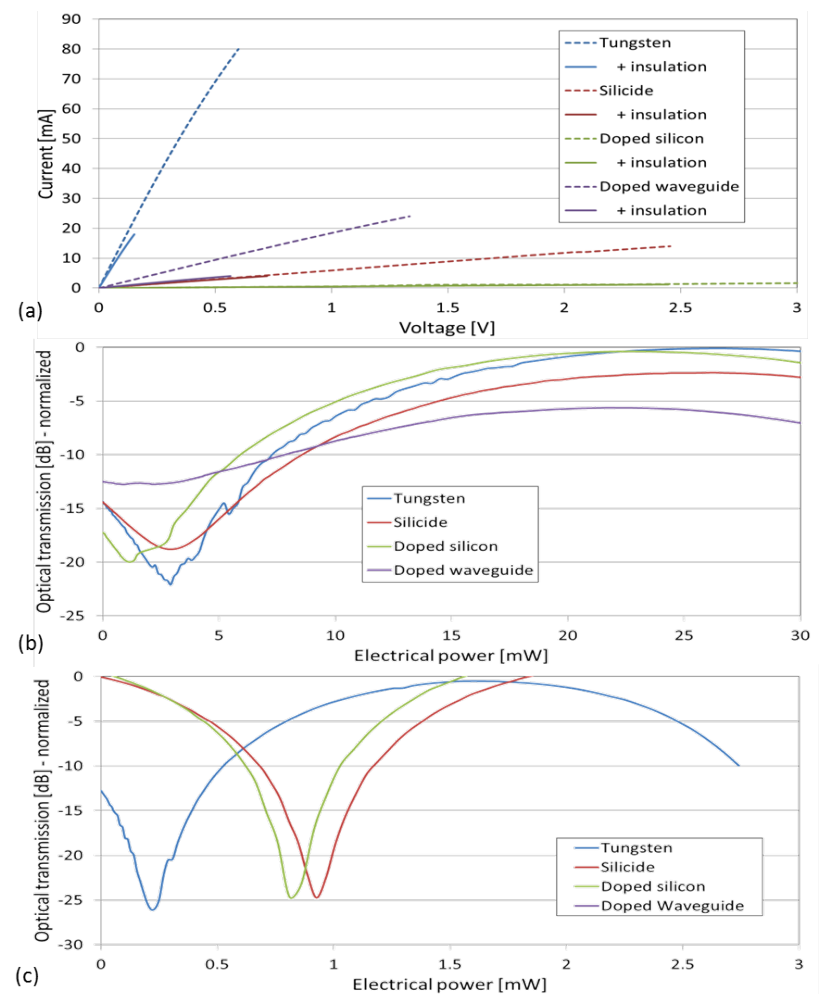

Fig. 3. Optical and electrical response of the heaters. (a) I-V curves (b) Optical transmission as function of the electrical power, without insulation trench, (c) same for heaters with insulation trench.

\section{B. Characterization method}

We characterized the heaters both electrically and optically. Light was coupled in the MZI through grating couplers. To measure the thermo-optic efficiency we applied a current sweep while monitoring the optical output at a fixed wavelength, as well as the voltage over the heater. The optical transmission follows a sinusoidal curve from which we can extract the thermo-optic phase responsivity. We also measured the dynamic response, and recorded both heating and cooling time of the optical response.
TABLE I. HEATER PERFORMANCE COMPARISON

\begin{tabular}{lcccc}
\hline Heater type & $\begin{array}{c}\text { Efficiency } \\
{[\mathrm{mW} / \pi]}\end{array}$ & $\begin{array}{c}\text { Line Resistivity } \\
{[\Omega / \mu m]}\end{array}$ & $\begin{array}{c}\text { Heating } \tau \\
1 / \mathrm{e}[\mu \mathrm{s}]\end{array}$ & $\begin{array}{c}\text { Cooling } \tau \\
1 / \mathrm{e}[\mu \mathrm{s}]\end{array}$ \\
\hline Without Insulation & & & & \\
Doped silicon & 20.4 & 293 & 21.3 & 66.0 \\
Silicide & 22.5 & 27.3 & 19.1 & 75.8 \\
Tungsten & 23.4 & 1.1 & 38.2 & 45.11 \\
Doped waveguide & 21.9 & $\mathrm{~N} / \mathrm{A}^{*}$ & 43.4 & 39.8 \\
\hline With Insulation & & & & \\
Doped silicon & 1.42 & 317 & 151 & 217 \\
Silicide & 1.49 & 28.0 & 188 & 218 \\
Tungsten & 1.42 & 1.3 & 198 & 227 \\
Doped waveguide & 1.30 & N/A* & 236 & 156 \\
\hline \multicolumn{5}{r}{} \\
\hline
\end{tabular}

\section{EXPERIMENTAL RESULTS}

Figure $3 \mathrm{a}$ shows the electrical I-V curve of the different heaters. The values for the line resistivity are calculated from the slope, and are also listed in table I. Figure $3 \mathrm{~b}$ and c show the phase response for the different heater types as a function of the electrical power. We clearly see the difference between heaters with and without the thermal insulation trenches. This confirms that the thermal environment is the dominant factor in determining the heater efficiency. The actual heater responsivity in $\mathrm{mW} / \pi$ phase shift for the different heaters is listed in Table I. The heaters with thermal insulation trenches and undercut are 10-20 times more efficient than without.

Table I also shows the heating time and cooling time of the different heaters. We see that the doped silicon and the silicide have quite short time constants, while tungsten heaters and the in-waveguide heaters have a somewhat higher time. This can be attributed to more heat spreading, causing a larger volume to heat up. For the heaters with insulation, the time constants are higher, due to lower coupling to the environment.

\section{CONCLUSION}

We have demonstrated and compared different heaters based on CMOS processes integrated in the same silicon photonic platform. We show that heaters have similar tuning efficiency, but cover two orders of magnitude in electrical properties, offering a large design freedom. When thermal insulation is applied, heater efficiency improves with a factor of 20 , but at the cost of a slower response time.

\section{ACKNOWLEDGMENT}

This work was supported in by imec's Optical IO industrial affiliation program.

\section{REFERENCES}

[1] P. Dong, W. Qian, H. Liang et al., "Thermally tunable silicon racetrack resonators with ultralow tuning power," Opt. Express, vol. 18, no. 19, pp. 20298-20304, 2010.

[2] J. Van Campenhout, W. M. J. Green, S. Assefa et al., "Integrated nisi waveguide heaters for cmos-compatible silicon thermooptic devices," Opt. Lett., vol. 35, no. 7, pp. 1013-1015, 2010.

[3] A. Masood, M. Pantouvaki, D. Goossens et al., "Cmos-compatible tungsten heaters for silicon photonic waveguides," in Group IV Photonics 2012, aug. 2012, pp. $234-236$.

[4] P. Verheyen, G. Lepage, J. Van Campenhout et al., "Co-integration of ge detectors and si modulators in an advanced si photonics platform," in SPIE Photonics Europe. International Society for Optics and Photonics, 2012, pp. $843114-843114$. 